Open Access

\title{
Rationale and design of the Adapted Physical Activity in advanced Pancreatic Cancer patients (APACaP) GERCOR (Groupe Coopérateur Multidisciplinaire en Oncologie) trial: study protocol for a randomized controlled trial
}

\author{
Cindy Neuzillet ${ }^{1 *}$, Mathieu Vergnault ${ }^{2}$, Franck Bonnetain ${ }^{3}$ and Pascal Hammel ${ }^{1}$
}

\begin{abstract}
Background: Exercise during chemotherapy is a promising strategy to reduce fatigue and improve health-related quality of life (HRQOL). It has been shown to be feasible and efficient in various cancers, including advanced-stage cancers. Effects of physical activity have never been explored in advanced pancreatic ductal adenocarcinoma (PDAC). We aim to evaluate the effects of an exercise intervention in this setting.

Methods: This randomized, national, multicenter, interventional study will examine the effectiveness of an unsupervised, home-based, 16-week adapted physical activity (APA) program. Specificities of advanced PDAC for the implementation of the APA program will be taken into account (healthy volunteer as physical activity partner instead of patient groups, nutritional management). The main inclusion criteria are: patients with histologically confirmed, unresectable PDAC; scheduled for chemotherapy; performance status 0-2; age $\geq 18$; and physical activity partner. In total, 200 patients will be randomized into either the APA program (aerobic and resistance exercises) in addition to usual care (including chemotherapy at the investigator's choice), or usual care. The primary objective will be the effect on fatigue (Multidimensional Fatigue Inventory, MFI-20) and HRQoL (European Organization for Research and Treatment of Cancer-Quality of Life-C30 questionnaire, EORTC-QLQ-C30; co-primary endpoint) at week 16. As secondary objectives, the effects of the exercise intervention on pain, anxiety, depression, nutritional status, insulin resistance, tolerance of chemotherapy, survival, and adherence to the APA program will be evaluated.

Discussion: Patients with advanced PDAC are strongly affected by fatigue, and are thus likely to benefit from an exercise intervention. Moreover, exercise may have a potential beneficial effect on tumor outcome by reducing insulin resistance and insulin/insulin-like growth factor-1 (IGF-1) secretion. However, an exercise intervention may appear challenging due to multiple PDAC-related symptoms such as fatigue, depression, pain, and denutrition. We hypothesize that an APA program taking into account specific characteristics of PDAC may improve symptoms and HRQoL. If demonstrated to be feasible and effective, such APA programs will be systematically proposed to patients with advanced PDAC in addition to usual care.
\end{abstract}

Trial registration: ClinicalTrial.gov Registration number: NCT02184663; Registration date: 2 July 2014.

Keywords: Fatigue, Health-related quality of life, Insulin resistance, Locally advanced, Metastatic, Pancreatic adenocarcinoma, Physical exercise, Study protocol, Supportive care, Survival

\footnotetext{
* Correspondence: cindy.neuzillet@orange.fr

'Digestive Oncology Department and UMR1149, Beaujon University Hospital,

Hôpitaux Universitaires Paris Nord Val de Seine (HUPNVS), Assistance

Publique-Hôpitaux de Paris (AP-HP), 92110 Clichy La Garenne, France

Full list of author information is available at the end of the article
}

C Biomed Central (c) 2015 NEUZILLET et al. Open Access This article is distributed under the terms of the Creative Commons Attribution 4.0 International License (http://creativecommons.org/licenses/by/4.0/), which permits unrestricted use, distribution, and reproduction in any medium, provided you give appropriate credit to the original author(s) and the source, provide a link to the Creative Commons license, and indicate if changes were made. The Creative Commons Public Domain Dedication waiver (http://creativecommons.org/publicdomain/zero/1.0/) applies to the data made available in this article, unless otherwise stated. 


\section{Background}

\section{Current knowledge about physical activity in cancer patients}

Physical activity is associated with a decreased risk of cancer, including colorectal, breast, and endometrial cancers [1-4]. According to the World Health Organization (WHO), 30 minutes of physical activity five times per week may reduce the risk of developing colorectal or breast cancer by about $25 \%$.

There is accumulating evidence that physical activity is also beneficial for patients with cancer in reducing disease and/or treatment-induced symptoms (including pain, fatigue, and anxiety/depression), and improving health-related quality of life (HRQoL) as well as treatment tolerance and adherence [5-8]. Fatigue in cancer patients is multifactorial and can be related to the disease itself, treatment, and bed rest, leading to deconditioning. Deconditioning, that is, loss of physical (cardiorespiratory function and muscle strength) and psychological fitness caused by reduced physical activity, is one of the main causes of cancer-related fatigue [9]. Paradoxically, rest may have deleterious effects on these patients, while physical exercise is the best way to reduce deconditioning and fatigue [10-12]. Several studies have reported a $30 \%$ reduction in cancer-related fatigue and maintained cardiorespiratory fitness and muscle strength with higher physical activity, even in advancedstage cancer [8].

A beneficial effect of physical activity on overall survival (OS) has also been reported in the most frequent cancers such as those of the breast, colon, and prostate [13]. Exercise reduces circulating estrogen and sex hormone binding globulin (SHBG) levels in sex hormonedependent cancers, improves insulin profile (insulin-related pathways including insulin-like growth factor 1 [IGF-1]), reduces inflammation, and increases natural killer and T-cell-mediated immunity [13-18]. Moreover, physical activity may improve survival through its impact on HRQoL, a prognostic indicator of survival in various cancers including pancreatic cancer $[19,20]$.

The French National Cancer Institute (INCa) (http:// www.afsos.fr/) promotes physical activity in the prevention and management of cancer at any time. The French association for supportive care in cancer (Association Francophone pour les Soins Oncologiques de Support, AFSOS, http://www.afsos.fr/), the American College of Sports Medicine (ACSM), and the American Cancer Society (ACS) have edited guidelines for "adapted physical activity" (APA) in cancer patients based on published data [21-24]. In France, but also in many other countries, adapted physical activity (APA) is a concept defined by the International Federation of Adapted Physical Activity (IFAPA) as " a cross-disciplinary body of practical and theoretical knowledge directed toward impairments, activity limitations, and participation restrictions in physical activity. It is a service delivery profession and an academic field of study that supports an attitude of acceptance of individual differences, advocates access to active lifestyles and sport, and promotes innovative and cooperative service delivery, supports, and empowerment. Adapted physical activity includes, but is not limited to, physical education, sport, recreation, dance, creative arts, nutrition, medicine, and rehabilitation." [25]. In the cancer setting, the term APA is used for exercise interventions involving professional trainers who have graduated with an academic degree in APA. Implementation of an APA program in patients with cancer implies a multidisciplinary collaboration between the cancer-care medical team and APA professionals. The APA program should be individualized according to the patient (physical fitness, exercise type preferences, psychological functions, and expectations), the cancer (stage, treatments, and tolerance), and the social environment. A combined aerobic exercise and resistance-training program is hypothesized to be the most efficient way to improve physical fitness and decrease fatigue and therefore should be favored [21-24]. Patient adherence to the APA program is crucial for its efficacy. Performing exercise in groups of patients having similar physical capabilities and under the supervision of an APA professional whenever possible is the best way to ensure patient motivation. The APA program also requires the contribution of nutrition specialists (dietitians or physicians) to balance energy intake with energy expenditure. Little is known about the optimal duration of exercise interventions, and no specific recommendation from evidence-based guidelines exists [24]. In the Cochrane meta-analysis review of randomized controlled trials evaluating the effectiveness of exercise interventions on health-related quality of life (HRQoL) in people with cancer during active treatment [7], the length of exercise intervention varied greatly between trials (3-26 weeks), with a modal duration of 12 weeks. Similarly, a modal duration of exercise interventions of 12 weeks was reported in the Cochrane meta-analysis of trials that investigated the effect of exercise on the management of cancerrelated fatigue [8]. In patients who exercised during treatment, improvements in global health and physical and emotional functioning compared to baseline values were found at week 12 [24]. Noticeably, in these studies, patients were allowed to continue exercise after the end of the intervention period.

\section{Epidemiology and management of pancreatic ductal adenocarcinoma (PDAC)}

Pancreatic ductal adenocarcinoma (PDAC) is the second cancer of the digestive tract in incidence and one of the poorest prognostic tumors with a 5-year survival rate of less than $5 \%$ [26]. More than $80 \%$ of patients have an 
advanced, unresectable disease at diagnosis. Therapeutic options remain limited in this setting [27]. Treatment consists mainly of chemotherapy with weekly (gemcitabine or gemcitabine plus nab-paclitaxel regimens) or biweekly regimens (fixed-dose rate [FDR] gemcitabine, the combinations of 5-fluorouracil, irinotecan, and oxaliplatin [FOLFIRINOX], or gemcitabine and oxaliplatin [GEMOX]). Radiation therapy is limited to selected patients with non-metastatic disease. To date, there are no data about the role of APA in patients treated for an advanced PDAC.

\section{Hypothesis for beneficial effects of exercise intervention in patients with advanced PDAC}

Fatigue is a common disease-related symptom in patients with advanced PDAC that contributes to impaired HRQoL [28, 29]. We hypothesize that physical exercise may limit physical deconditioning and reduce fatigue in these patients. Physical activity may have a beneficial effect on pain, anxiety, and depression, symptoms affecting more than half of PDAC patients, and may result in a global improvement of HRQoL and in delayed deterioration of HRQoL [30]. Moreover, an improvement in HRQoL may translate into a survival benefit [20].

There is consistent evidence on the relationship between insulin resistance, insulin and IGF-1 secretions, and pancreatic carcinogenesis [31-37]:

- Obesity and type II diabetes (metabolic syndrome) are risk factors for PDAC (relative risk $[R R]=1.2-1.6$ and $1.8-2.0$, respectively) and physical activity may have a protective effect $(R R=0.72)$,

- Metformin, a drug that decreases insulin resistance, reduces the risk of developing PDAC in in vivo preclinical studies and in observational clinical studies of patients with type II diabetes,

- Insulin receptor is overexpressed in PDAC, and insulin and IGF-1 stimulate proliferation of PDAC cells in vitro,

- Some polymorphisms of IGF, IGF receptor, and IGF-binding protein are associated with an increased risk of PDAC and shorter survival in PDAC patients,

- Weight loss, impaired glucose tolerance and diabetes, along with peripheral insulin resistance are frequently observed in PDAC patients.

Physical exercise has been associated with reduced insulin resistance and insulin and IGF-1 secretions [13]. In addition, PDAC usually causes an intense inflammatory reaction that contributes to tumor-related cachexia [38]. Exercise can modulate this process and may have a beneficial effect on disease outcome (progression-free survival [PFS] and OS) in patients with advanced PDAC.

\section{Specificities of advanced PDAC affecting the implementation of the APA program}

Sessions in groups are suitable to maintain patient motivation, particularly in an adjuvant setting (for example, for breast and colon cancer). In women with breast cancer, being part of a supportive social environment is acknowledged in motivational and behavioral theories as a key factor to health behavior change and stands among the strongest incentives for exercise motivation [39]. However, we believe that patients with advanced PDAC are not suitable for group sessions. These patients have a poor prognosis and can deteriorate during the exercise intervention period. Therefore, regular meetings of patients treated for PDAC may lead to negative and counterproductive psychological impact on the group. For this reason, we propose to include a "physical activity partner," a healthy volunteer, chosen by the patient, to attend the exercise sessions. This practice is expected to sustain the patient motivation and to be beneficial for the partner in terms of psychological outcome, while avoiding the potentially deleterious psychological effects of the patient group [40]. Such organization will imply a psychological evaluation of the partner to verify that he/ she is not experiencing psychological burnout.

Patients with PDAC often suffer from weight loss due to an inadequate dietary intake combined with increased energy expenditure [41]. Denutrition is experienced by $70-80 \%$ of PDAC patients and is multifactorial in origin including inflammatory and hypercatabolic syndrome, stenosis of the digestive tract, cholestasis, exocrine pancreatic insufficiency, diabetes, anxiety, depression, and chemotherapy adverse effects (nausea/vomiting, mucitis, diarrhea, and loss of appetite). Patients with PDAC appeared to have a significantly increased basal resting energy expenditure (REE) and spontaneously reduced physical activity level compared with healthy individuals [41]. As the APA program is expected to increase energy expenditures, nutritional management will be crucial for monitoring and adapting food intakes to ensure that patients meet their nutritional needs.

There is consistent evidence that a 12-week intervention is the minimal duration of exercise intervention to improve HRQoL and fatigue in patients with cancer [7, $8,24]$. In the setting of pancreatic ductal adenocarcinoma (PDAC), median progression-free survival (PFS) in patients with metastatic disease ranges from 3 to 4 months with gemcitabine, from 5 to 6 months with gemcitabine plus $n a b$-paclitaxel or the FOLFIRINOX combination regimens, and from 9 to 10 months in patients with locally advanced PDAC [42-44]. Deterioration of HRQoL is closely related to disease progression and survival, and can be affected by a change in chemotherapy regimen in the case of tumor progression [20]. Thus, evaluation of HRQoL can be biased in many ways by 
disease progression. Therefore, we propose a 16-week intervention and HRQoL and fatigue data evaluation at week 16, since most patients are expected to remain free of disease progression during this time period and to remain on the same chemotherapy regimen. In addition, evaluation of tumor status under treatment in advanced PDAC is usually performed every 8 weeks; thus, this length of exercise intervention was judged as well suited for the timing of tumor evaluation.

\section{Methods/design}

\section{Study objectives and hypotheses}

The APACaP (Activité Physique Adaptée et chez des patients ayant un Cancer du Pancréas non résécable; adapted physical activity in patients with unresectable pancreatic adenocarcinoma) study aims to assess the efficacy of an unsupervised 16-week APA program on physical fatigue (Multidimensional Fatigue Inventory, MFI-20) and targeted dimensions of HRQoL (global QoL, fatigue, physical functioning, and pain; European Organization for Research and Treatment of CancerQuality of Life-C30 questionnaire, EORTC-QLQ-C30), unified as the co-primary endpoint (single sufficient design) at the end of the intervention in advanced PDAC patients. Secondary objectives include assessing the beneficial effects on pain, other HRQoL dimensions, depression, nutritional status, insulin resistance, cancertreatment tolerance, PFS, and OS.

\section{Design}

The APACaP study is designed as a national, prospective, multicenter, randomized, controlled trial to test the efficacy of an APA program, in which patients will be allocated either to the intervention group invited to perform exercise in addition to usual care, or to the usual care control group (Fig. 1). Because blinding of participants toward allocation is not feasible, this study will have an open-label design.

\section{Patients}

Patients with histologically confirmed, previously untreated, unresectable (locally advanced or metastatic) PDAC who meet the following criteria will be eligible for the APACaP study: scheduled for chemotherapy; Eastern Cooperative Oncology Group (ECOG) performance status (PS) $\leq 2$ (Additional file 1: Table S1); age $\geq 18$ years; at least one bi-dimensionally measurable lesion according to the Response Evaluation Criteria In Solid Tumors (RECIST) v1.1 criteria [45]; designated physical activity partner; signed and dated informed consent; and registration in a national health care system (CMU included). Patients will be excluded if their cardiovascular, respiratory, psychiatric, musculoskeletal, or neurological condition contraindicates exercise practice. Participants of other clinical studies will be eligible for the APACaP study if the primary endpoints of both studies do not overlap.

\section{Ethics and regulatory considerations}

Written informed consent will be obtained from all patients. The current study is to be conducted in accordance with globally accepted standards of Good Clinical Practice (ICH-E6), European Directive 2001/20/EC, and the revised version of the Declaration of Helsinki set out in the European Directive, as well as with the Code de Santé Publique specific to France. The protocol has been submitted for formal approval of the study conduct to ensure that the study meets the local regulations to the Health Authorities, that is, the Agence Nationale de Sécurité du Médicament et des produits de santé (ANSM), and to a properly constituted Ethics Committee. The study was approved by Health Authorities (ANSM), and full ethical approval was obtained from a unique Ethics Committee for all centers involved (CPP Ile-de-France VI N 32-14; ID RCB: 2014A00228-39), in compliance with French regulations. The study has been registered on ClinicalTrial.gov (NCT).

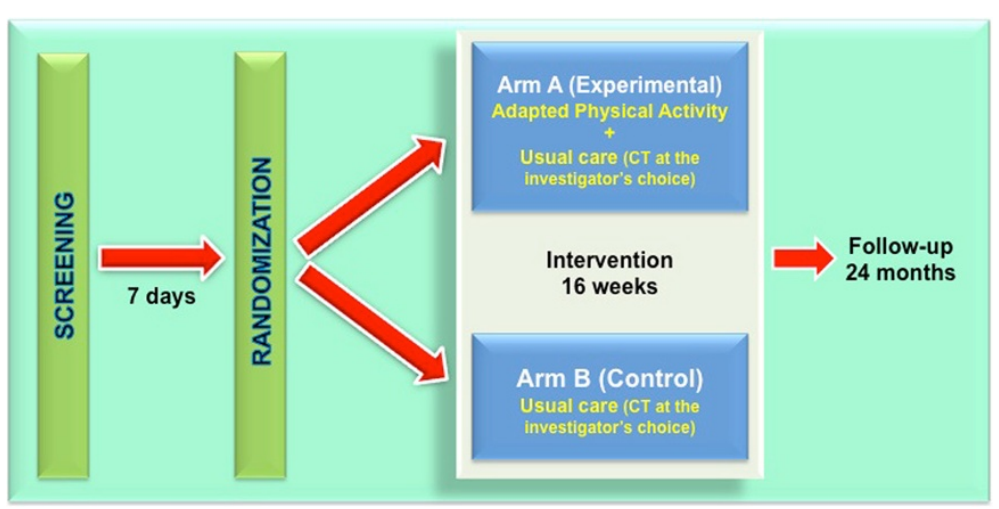

Fig. 1 The APACaP study design. CT: chemotherapy 


\section{Recruitment and randomization}

This study is promoted by the Groupe Coopérateur Multidisciplinaire en Oncologie (GERCOR) and will be conducted in 16 centers in France (managed by the GERCOR and Partenariat de Recherche en Oncologie DIGEstive [PRODIGE] cooperative groups). Newly diagnosed patients with advanced PDAC identified as eligible will be informed about the program and invited to participate in the study during a regular outpatient visit.

The clinician will assess the patient eligibility and will provide each patient and/or a legal representative with relevant, comprehensive, verbal and written information regarding the objectives and procedures of the study as well as their possible risks. The patient will be given a 1week reflection period (up until the first chemotherapy cycle) in order to inquire about study details. All questions raised by the patient should be answered in a satisfying manner. The investigator will inform each patient about his/her right to withdraw from the study at any time for any reason. A signed informed consent will be obtained from each patient and/or legal representative prior to undertaking any study-related procedure.

Eligible patients will be randomized in a 1:1 ratio by a central computer-assisted procedure centralized at the GERCOR data center. Randomization will be performed using a minimization technique and will be stratified per center, cancer stage (locally advanced versus metastatic), chemotherapy schedule (weekly versus biweekly regimen), ECOG PS (0-1 versus 2), and baseline physical activity level (low, moderate, or intense according to the Global Physical Activity Questionnaire [GPAQ]). Each patient who enters into the study will receive a unique subject identification number.

\section{Study protocol Control group}

Participants assigned to the control group will receive usual care (that is, with no physical activity intervention) including chemotherapy at the investigators' choice (according to the patient general condition and biology, comorbidity, and tumor stage: weekly gemcitabine or gemcitabine plus nab-paclitaxel regimens; biweekly FDR gemcitabine, FOLFIRINOX, or GEMOX), outpatient clinical visits according to the regular schedule, nutritional support according to the national recommendations (Société Francophone de Nutrition Clinique et Métabolisme [SFNEP]), and tumor evaluation based on carbohydrate antigen 19-9 (CA 19-9) serum levels and thoraco-abdominopelvic computed tomography (TAP-CT) every 8 weeks.

\section{Intervention group}

Participants assigned to the intervention group will receive usual care plus a 16-week APA program.
The APA program, designed according to the AFSOS recommendations, will be directed by an APA professional trainer selected from a list of certified professionals chosen for the study. Exercise sessions will be implemented in addition to daily life activities. The exercise training program will consist of one supervised demonstration session with the APA professional, and thereafter the training will be continued as unsupervised, home-based exercise training sessions.

Two types of exercises will be proposed: 1) aerobic exercises including walking, and/or Nordic walking, and/or cycling, according to patient preference and 2) resistance exercises using elastic bands to maintain or increase arm and leg muscle strength.

Duration, frequency, and intensity of exercises will be adapted for each patient by the APA professional according to standardized guidelines. Patient fitness before treatment (estimated by the GPAQ), deconditioning level, and baseline physical evaluation (6-minute walking test and maximal strength test) will be considered. The APA professional will define exercise intensity based on baseline patient physical evaluation. Aerobic exercises will be performed with an intensity that will allow the patient to speak comfortably. The muscle-strengthening program will consist of repetitions performed at $50 \%$ of baseline maximal strength test. One-repetition maximum (1-RM) is a measure of muscular strength; it is the highest weight that can be lifted for one repetition and one repetition only. Elastic bands (TheraBands ${ }^{\circ}$ ), available as the eight color-coded resistance level bands, are frequently used to assess the 1-RM [46, 47]. The APA professional will measure the distance from starting position to ending position during exercise with the elastic band to determine its deformation. After a warm-up without the elastic band and based on the APA professional assumption of the patient's approximate strength, the appropriate band will be chosen for exercise. If the patient can perform more than ten repetitions with good form, a higher resistance band will be tested. The amount of deformation, the elastic band color, and the number of repetitions are required parameters to calculate a 1-RM. The 1-RM test is calculated based on the Brzycki equation: $1-\mathrm{RM}=$ weight loaded/ $(1,0278$ $(0,0278 \times$ number of repetitions) $)$ [48]. The weight loaded is defined for each color of elastic bands depending on the level of deformation [49].

Physical activity sessions will comprise exercises for warming up followed by aerobic or resistance exercises and a recuperation period. The warming-up and recuperation periods will account for at least $30 \%$ of the session duration. The aim of the APA program is to gradually reach a total of 30 minutes of aerobic training, three to five times per week (according to physical condition), and to perform strengthening activities at least 
two times per week for all the major muscle groups, excluding the warming-up and recuperation periods. A lower intensity activity week will be planned every 4 weeks to prevent patient exhaustion.

Patients will be asked to report on their APA program in a dedicated activity booklet. Photographs and explanations for good positioning and proper execution of exercises will be provided to patients. The APA professional will monitor weekly the program tolerance and adherence during a video call (a webcam will be provided to the patient if necessary). The booklet can be used to help patients recall exercises performed and difficulties encountered. The video call will allow the APA professional to evaluate leisure activities, sessions of aerobic training, and strengthening exercises, and to assess the patient tolerance and difficulties in completing the physical activity level. Depending on this evaluation, the APA program will be adapted according to the scheme illustrated by Fig. 2. Difficulty of activities will be increased in duration, then in frequency, and ultimately in intensity. Exercise intensity will be enhanced only if the patient tolerates an increase in duration and frequency.

A supervised session with elastics can be performed during video call on patient demand.

After completion of the 16-week study program, the patient will be offered the option of pursuing the physical exercises.
The APA program will be temporary interrupted in the following cases: uncontrolled infection, thromboembolic event (first 7 days of anticoagulant therapy), uncontrolled pain, symptomatic anemia or hemoglobin level $<10 \mathrm{~g} / \mathrm{dl}$, platelet counts $<50,000 / \mathrm{mm}^{3}$, and a major surgery.

Termination of the patient participation in the study can occur in the following situations: on patient's request, deterioration of general condition with ECOG PS $>2$, uncontrolled symptoms, clinical problem definitively contraindicating exercise practice, clinicians' or trainers' decision in the best interest of the patient, serious adverse event (AE) resulting from physical exercise or preventing the patient from pursuing exercise, loss to follow-up, and major protocol deviation.

The patient will be asked to designate a physical activity partner (a healthy family member/relative or friend) who can attend (without obligation of performing exercises) and support the patient for at least one exercise session per week. The physical activity partner will be given all needed information and will be allowed to attend the patient visits with the physical activity trainer. Psychological follow-up of the physical activity partner will be scheduled using the Hospital Anxiety and Depression Scale (HADS).

The APA program will be associated with a nutritional intervention supervised by a dietitian. Nutritional management will be designed according to the SFNEP

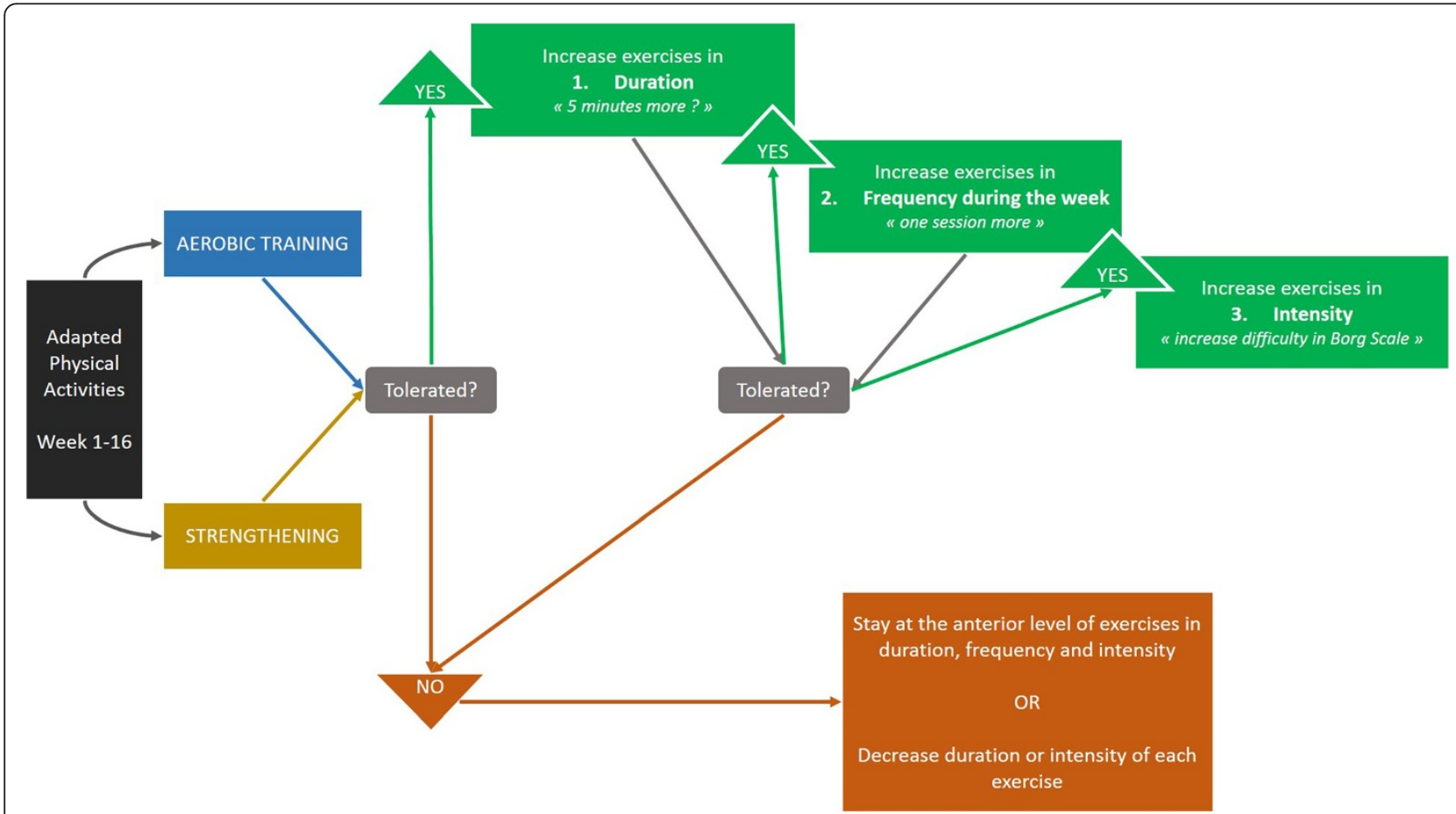

Fig. 2 Mapping of the progression steps of the 16-week adapted physical activity (APA) program 
recommendations. Practically, patient nutritional status will be assessed based on clinical (actual weight, weight loss, and body mass index) and biological (albumin, prealbumin, and C-reactive protein [CRP]) parameters. The daily calorie and protein requirements will be calculated using the Harris-Benedict equation for patient basal energy expenditures. To determinate the total daily caloric requirements, two variables, the individual cancerrelated stress factor (REE $\times 1.3$ ) and the activity factor (REE x 1.4 a 1.6) will be considered. A three-day food intake evaluation will be performed. Nutritional intervention (oral feeding and enteral and/or parenteral nutrition) will be decided according to the SNFEP guidelines.

\section{Safety}

All AEs reported by the patient or observed by the clinician and the APA professional (sports accidents, exhaustion, or injuries) will be recorded. All serious AEs will be reported to the ANSM and to the accredited European Commission that approved the study protocol.

It will be the responsibility of the clinician investigator to record all the relevant information regarding any event occurring during the APA program. The investigator will be requested to assess the relationship between the intervention and the occurrence of each $\mathrm{AE}$ using clinical criteria. Alternative causes such as natural history of the underlying diseases, concomitant therapy, other risk factors, and the temporal relationship of an event to the intervention will be considered and investigated.

The investigators and all other appropriate persons will be informed of findings that could adversely affect the safety of patients in a timely manner during the study duration.

\section{Study endpoints and measures Primary outcome}

The primary outcome of the APACaP study is to assess the effects of the APA program on fatigue (one targeted dimension) and HRQoL (four targeted dimensions), unified as a co-primary endpoint, at the end of a 16-week intervention in patients with advanced PDAC. We hypothesize that exercise intervention will significantly improve at least one of these five parameters without significantly deteriorating the others.

Fatigue will be measured using the validated French version of the MFI-20 questionnaire [50, 51]. The MFI20 is a 20-item self-reported instrument, organized into five scales: general, physical, mental fatigue, and reduced activity and motivation, and is designed to assess multiple fatigue dimensions. The targeted dimension will be the physical fatigue scale. A change of two or more units will be considered as a clinically relevant change [52].
The EORTC-QLQ-C30 questionnaire comprising five functional scales (physical, role, emotional, cognitive, and social), one global QoL scale, and a 10-item symptom scale (including fatigue and pain) will be used to measure HRQoL [53]. The four targeted dimensions will be global QoL, fatigue, physical functioning, and pain. A change of five or more units will be considered as the minimal clinically important change [54].

\section{Secondary outcomes}

Secondary outcomes will consist in the longitudinal analysis of fatigue and HRQoL, and the effects on pain, depression, nutritional status, insulin resistance, cancertreatment tolerance, and survival.

Longitudinal analysis of fatigue and HRQoL will be performed using a linear mixed-effects regression model (repeated measures analysis of variance [ANOVA]) to test randomization and time effects and a timetreatment interaction. In case of non-random missing data, pattern mixture models will be used. Time until definitive deterioration (TUDD) of targeted scores will be defined as the interval between the measurement of HRQoL at baseline (before randomization) and the date of occurrence of a 5-point or greater HRQoL score deterioration, no improvement thereafter, or death from all causes $[20,55,56]$. If deterioration is not observed, patients will be censored at the last date at which the patient has filled out the questionnaire.

Pain will be assessed using a visual analogue scale (VAS), analgesics consumption, and the French validated version of the Brief Pain Inventory-Short Form (BPI-SF) questionnaire $[57,58]$. The BPI-SF questionnaire is a 15item scale evaluating five parameters of pain: severity, impact on daily function, location, pain medications, and amount of pain relief in the past 24 hours or the past week. Two scores will be calculated to assess the severity of pain and the impact of pain on daily functions.

Anxiety and depression will be rated using the French validated version of the HADS questionnaire and based on anxiolytic and antidepressant consumption [59, 60]. The HADS questionnaire evaluates emotional state reported by the patient during the week before the assessment date. It consists of 14 items including seven for anxiety and seven for depression. Each item is rated on a $0-3$ rating scale $(3=$ highest intensity level of feeling). The anxiety and depression scores will be calculated by the sum of the corresponding seven items. A score $>11$ will be considered as significant for the presence of an anxiety or depression problem.

Nutritional status will be evaluated based on weight, body mass index $\left(\mathrm{kg} / \mathrm{m}^{2}\right)$, body composition (bioimpedancemetry method in equipped centers), food intake (assessed using a VAS), and biological parameters (albumin, prealbumin, and CRP). 
Insulin resistance will be determined based on levels of fasting plasma glucose, insulin, hemoglobin A1c (HbA1c hemoglobin), plasma IGF-1, insulin and antidiabetic agent requirements, and homeostasis model assessment (HOMA).

Tolerability of chemotherapy will be assessed in terms of toxicities or treatment delay. Toxicities will be graded using the Common Terminology Criteria for Adverse Events (CTCAE) v 4.0.

Effects on PFS and OS will be analyzed. PFS is defined as the time elapsed from treatment initiation to first tumor progression (according to the RECIST v1.1 criteria), or death from any cause; censored are patients alive or lost to follow-up $[45,56]$. OS is defined as the time elapsed from treatment initiation to death from any cause, or last follow-up.

Exercise tolerance will be monitored using the booklet, visual scales (dyspnea, muscular pain), and a Borg scale. The effects of physical activity on deconditioning, cardiorespiratory function, and muscle strength will be evaluated using the International Physical Activity Questionnaire (IPAQ), the resting heart rate and blood pressure, a 6-minute walking test with monitoring of the heart rate during exertion, a maximal strength test with elastic bands, and bioimpedancemetry. Adherence to the APA program will be registered in the patient booklet and during video calls.

Data will be collected for the 24-month follow-up period after enrollment. Tables 1 and 2 provide a summary of the study activities, measures, and schedule of visits.

\section{Sample size}

To detect a difference between the two groups at a global type I error of $5 \%: 1$ ) a change of five units (standard deviation $[S D]=10)$ for at least one of the EORTC-QLQC30 targeted dimensions (global QoL, fatigue, physical functioning, and pain) with an alpha error risk of $1 \%$ in a bilateral design (with a Bonferroni adjustment of $4 \% / 4=1 \%$ ) and a statistical power of $80 \%$ with a mean comparison test, a total of 190 patients are required [54]; 2) or a change of two units $(\mathrm{SD}=4)$ for the targeted dimension of the MFI-20 scale (physical fatigue), with an alpha error risk of $1 \%$ in a bilateral design (with a Bonferroni adjustment of $1 \%$ ) and a power of $80 \%$ with a mean comparison test, a total of 190 patients are required [52].

The effects of exercise on fatigue and HRQoL will be tested after 16 weeks, 6 months, and 24 months postenrollment. An interim analysis will be performed after half of the patients have achieved at least 6 months of follow-up using the O'Brien-Fleming method (to reject the null hypothesis [H0; efficacy] or to reject the study hypothesis [H1; futility]). All analysis will be performed using EAST software v5.
An arm will be considered superior to the other arm if at least one of the targeted dimensions of the EORTCQLQ-C30 is significantly superior without any targeted dimension being superior in the other arm. Otherwise, at least three out of the five targeted dimensions will be required to be significantly superior in order to consider that one of the study arms is globally superior to another arm.

To anticipate a dropout of $5 \%$ (that is, discontinuation of participation and loss to follow-up), a total of 200 patients will be randomized. In order to show that median TUDD for one of the targeted dimensions of the EORTC-QLQ-C30 shows change from 5 months to 8.5 months $(\mathrm{HR}=0.58)$ with an alpha error risk of $1 \%$ in a bilateral design (with a Bonferroni adjustment of $5 \% / 5=1 \%$ ) and a power of $80 \%$, a total of 200 patients with a minimal 12-month follow-up will be required to observe 170 events [20].

\section{Data analysis}

Descriptive statistics will be used to characterize the study population and the instrument scores at baseline and at follow-up. Statistical analyses will be performed with SAS v9.2, Stata v12, or R v2.15. All primary statistical analyses will be conducted on the intention-to-treat population. A $P$ value of $<0.01$ and of $<0.05$ will be considered statistically significant for the primary endpoint and secondary endpoints, respectively. Results will be presented for the total study population and according to center, cancer stage, chemotherapy schedule, ECOG PS, and baseline physical activity level.

The study data analysis will be conducted according to the following steps:

1. Description of the study sample Patient characteristics will be described for all the demographic and clinical data recorded at inclusion. Qualitative variables will be presented using absolute numbers and percentages, together with their $95 \%$ confidence intervals (CI). Quantitative variables will be reported using mean $( \pm \mathrm{SD})$ or median $(\min / \mathrm{max})$. Interaction tests will be performed. The comparison of means will be carried out using Student's $t$-test, the Wilcoxon rank-sum test, analysis of variance (ANOVA), and the Kruskall-Wallis test, as appropriate. The association of qualitative variables will be carried out using chi-square or Fisher's exact tests, as appropriate.

2. Score calculation and management of missing data Questionnaire scores will be calculated according to the authors' recommendations. Patients with missing data will be excluded from analysis. If data are missing at random, the average of items answered in the dimension will be measured, and if data are not missing at random, a multiple imputation method will be used to complete the dataset. 
Table 1 Schedule of visits and measures in the control arm

\begin{tabular}{|c|c|c|c|c|c|c|c|c|c|c|c|c|c|c|c|c|c|c|c|c|c|}
\hline & \multirow{2}{*}{$\begin{array}{l}\text { Screening visit } \\
\text { D7 }\end{array}$} & \multirow{2}{*}{$\begin{array}{l}\text { Randomization visit } \\
\text { D0 }\end{array}$} & \multicolumn{7}{|c|}{ Study visit } & \multirow{2}{*}{$\begin{array}{l}\text { End of treatment } \\
\text { W16 }\end{array}$} & \multicolumn{11}{|c|}{ Follow-up visit } \\
\hline & & & W2 & W4 & W6 & W8 & W10 & W12 & W14 & & W20 & M6 & M8 & M10 & M12 & M14 & M16 & M18 & M20 & M22 & M24 \\
\hline Informed consent & $x$ & & & & & & & & & & & & & & & & & & & & \\
\hline Randomization & & $x$ & & & & & & & & & & & & & & & & & & & \\
\hline Inclusion criteria & $x$ & & & & & & & & & & & & & & & & & & & & \\
\hline Physical activity partner & $x$ & & & & & & & & & & & & & & & & & & & & \\
\hline Physical activity level GPAQ & $x$ & & & & & & & & & & & & & & & & & & & & \\
\hline Demographic and clinical data & $x$ & & & & & & & & & & & & & & & & & & & & \\
\hline Concomitant treatment & $x$ & $x$ & $x$ & $x$ & $x$ & $x$ & $x$ & $x$ & $x$ & $x$ & $x$ & $x$ & $x$ & $x$ & $x$ & $x$ & $x$ & $x$ & $x$ & $x$ & $x$ \\
\hline Pathological data & $x$ & & & & & & & & & & & & & & & & & & & & \\
\hline Clinician visit & $x$ & $x$ & & & & $x$ & & & & $x$ & & $x$ & $x$ & $x$ & $x$ & $x$ & $x$ & $x$ & $x$ & $x$ & $x$ \\
\hline Physical exam & $x$ & $x$ & $x$ & $x$ & $x$ & $x$ & $x$ & $x$ & $x$ & $x$ & $x$ & $x$ & $x$ & $x$ & $x$ & $x$ & $x$ & $x$ & $x$ & $x$ & $x$ \\
\hline ECG & $x$ & & & & & & & & & & & & & & & & & & & & \\
\hline Questionnaires & & $x$ & & & & $x$ & & & & $x$ & $x$ & $x$ & $x$ & $x$ & $x$ & $x$ & $x$ & $x$ & $x$ & $x$ & $x$ \\
\hline 6-minute walking test & & $x$ & & & & $x$ & & & & $x$ & & $x$ & & & $x$ & & & $x$ & & & $x$ \\
\hline Maximal strength test & & $x$ & & & & $x$ & & & & $x$ & & $x$ & & & $x$ & & & $x$ & & & $x$ \\
\hline Body composition & & $x$ & & & & $x$ & & & & $x$ & & $x$ & & & $x$ & & & $x$ & & & $x$ \\
\hline Physical activity level IPAQ & & $x$ & & & & $x$ & & & & $x$ & & $x$ & & & $x$ & & & $x$ & & & $x$ \\
\hline \multicolumn{22}{|l|}{ Biology tests } \\
\hline Type 1 & & $x$ & & & & $x$ & & & & $x$ & & $x$ & & & $x$ & & & $x$ & & & $x$ \\
\hline Type 2 & $x$ & & $x$ & & $x$ & & $x$ & & $x$ & & $x$ & & $x$ & $x$ & & $x$ & $x$ & & $x$ & $x$ & \\
\hline Type 3 & & & & $x$ & & & & $x$ & & & & & & & & & & & & & \\
\hline Biobank (optional) & & $x$ & & & & & & & & & & & & & & & & & & & \\
\hline TAP-CT & $x$ & & & & & $x^{a}$ & & & & $x$ & & $x$ & $x$ & $x$ & $x$ & $x$ & $x$ & $x$ & $x$ & $x$ & $x$ \\
\hline CA-19.9 & $x$ & & & & & $x^{a}$ & & & & $x$ & & $x$ & $x$ & $x$ & $x$ & $x$ & $x$ & $x$ & $x$ & $x$ & $x$ \\
\hline Adverse events & & & $x$ & $x$ & $x$ & $x$ & $x$ & $x$ & $x$ & $x$ & $x$ & $x$ & $x$ & $x$ & $x$ & $x$ & $x$ & $x$ & $x$ & $x$ & $x$ \\
\hline
\end{tabular}

CA 19-9 carbohydrate antigen 19-9, CRP C-reactive protein, GPAQ Global Physical Activity Questionnaire, HbA1C glycated hemoglobin, HOMA homeostasis model assessment, IGF-1 insulin-like growth factor 1, IPAQ International Physical Activity Questionnaire, TAP-CT thoraco-abdominopelvic computed tomography

${ }^{\mathrm{a}} \mathrm{A}+/-2$-week time interval is considered as acceptable

Biology tests - type 1: blood cell count, CRP, fasting glucose, HbA1c, insulin/HOMA, IGF-1, albumin, prealbumin

Biology tests - type 2: blood cell count, CRP, fasting glucose, albumin, prealbumin

Biology tests - type 3: blood cell count, CRP, fasting glucose, insulin/HOMA, IGF-1, albumin, prealbumin

$\mathrm{X}$ : to be performed at this timepoint 
Table 2 Schedule of visits and measures in the intervention arm

\begin{tabular}{|c|c|c|c|c|c|c|c|c|c|c|c|c|c|c|c|c|c|c|c|c|c|}
\hline & \multirow{2}{*}{$\begin{array}{l}\text { Screening visit } \\
\text { D-7 }\end{array}$} & \multirow{2}{*}{$\begin{array}{l}\text { Randomization visit } \\
\text { D0 }\end{array}$} & \multicolumn{7}{|c|}{ Study visit } & \multirow{2}{*}{$\begin{array}{l}\text { End of treatment } \\
\text { W16 }\end{array}$} & \multicolumn{11}{|c|}{ Follow-up visit } \\
\hline & & & W2 & W4 & W6 & W8 & W10 & W12 & W14 & & W20 & M6 & M8 & M10 & M12 & M14 & M16 & M18 & M20 & M22 & M24 \\
\hline Informed consent & $x$ & & & & & & & & & & & & & & & & & & & & \\
\hline Randomization & & $x$ & & & & & & & & & & & & & & & & & & & \\
\hline Inclusion criteria & $x$ & & & & & & & & & & & & & & & & & & & & \\
\hline Physical activity partner & $x$ & & & & & & & & & & & & & & & & & & & & \\
\hline Physical activity level GPAQ & $x$ & & & & & & & & & & & & & & & & & & & & \\
\hline Demographic and clinical data & $x$ & & & & & & & & & & & & & & & & & & & & \\
\hline Concomitant treatment & $x$ & $x$ & $x$ & $x$ & $x$ & $x$ & $x$ & $x$ & $x$ & $x$ & $x$ & $x$ & $x$ & $x$ & $x$ & $x$ & $x$ & $x$ & $x$ & $x$ & $x$ \\
\hline Pathological data & $x$ & & & & & & & & & & & & & & & & & & & & \\
\hline Clinician visit & $x$ & $x$ & & $x$ & & $x$ & & & & $x$ & & $x$ & $x$ & $x$ & $x$ & $x$ & $x$ & $x$ & $x$ & $x$ & $x$ \\
\hline APA professional & & $x$ & & & & & & & & $x$ & & $(X)$ & & & $(X)$ & & & $(X)$ & & & $(X)$ \\
\hline Dietitian & & $x$ & $x$ & $x$ & & $x$ & & $x$ & & $x$ & & $(X)$ & & & $(X)$ & & & $(X)$ & & & $(X)$ \\
\hline Physical exam & $x$ & $x$ & $x$ & $x$ & $x$ & $x$ & $x$ & $x$ & $x$ & $x$ & $x$ & $x$ & $x$ & $x$ & $x$ & $x$ & $x$ & $x$ & $x$ & $x$ & $x$ \\
\hline ECG & $x$ & & & & & & & & & & & & & & & & & & & & \\
\hline Questionnaires & & $x$ & & & & $x$ & & & & $x$ & $x$ & $x$ & $x$ & $x$ & $x$ & $x$ & $x$ & $x$ & $x$ & $x$ & $x$ \\
\hline 6-minute walking test & & $x$ & & & & $x$ & & & & $x$ & & $x$ & & & $x$ & & & $x$ & & & $x$ \\
\hline Maximal strength test & & $x$ & & & & $x$ & & & & $x$ & & $x$ & & & $x$ & & & $x$ & & & $x$ \\
\hline Body composition & & $x$ & & & & $x$ & & & & $x$ & & $x$ & & & $x$ & & & $x$ & & & $x$ \\
\hline Physical activity level IPAQ & & $x$ & & & & $x$ & & & & $x$ & & $x$ & & & $x$ & & & $x$ & & & $x$ \\
\hline \multicolumn{22}{|l|}{ Biology tests } \\
\hline Type 1 & & $x$ & & & & $x$ & & & & $x$ & & $x$ & & & $x$ & & & $x$ & & & $x$ \\
\hline Type 2 & $x$ & & $x$ & & $x$ & & $x$ & & $x$ & & $x$ & & $x$ & $x$ & & $x$ & $x$ & & $x$ & $x$ & \\
\hline Type 3 & & & & $x$ & & & & $x$ & & & & & & & & & & & & & \\
\hline Biobank (optional) & & $x$ & & & & & & & & & & & & & & & & & & & \\
\hline TAP-CT & $x$ & & & & & $x^{a}$ & & & & $x$ & & $x$ & $x$ & $x$ & $x$ & $x$ & $x$ & $x$ & $x$ & $x$ & $x$ \\
\hline CA-19.9 & $x$ & & & & & $x^{a}$ & & & & $x$ & & $x$ & $x$ & $x$ & $x$ & $x$ & $x$ & $x$ & $x$ & $x$ & $x$ \\
\hline Adverse events & & & $x$ & $x$ & $x$ & $x$ & $x$ & $x$ & $x$ & $x$ & $x$ & $x$ & $x$ & $x$ & $x$ & $x$ & $x$ & $x$ & $x$ & $x$ & $x$ \\
\hline
\end{tabular}

CA 19-9 carbohydrate antigen 19-9, CRP C-reactive protein, GPAO Global Physical Activity Questionnaire, HbA1c glycated hemoglobin, HOMA homeostasis model assessment, IGF-1 insulin-like growth factor 1, IPAQ International Physical Activity Questionnaire, TAP-CT thoraco-abdominopelvic computed tomography

International Physical Activity Questionnaire, TAP-CT thora
aA 2-week time (+/-) interval is considered as acceptable

(X): only for patients pursuing the APA program after week 16

(X): only for patients punsung the APA program after week 16 , insulin/HOMA, IGF-1, albumin, prealbumin

Biology tests - type 2: blood cell count, CRP, fasting glucose, albumin, prealbumin

Biology tests - type 3: blood cell count, CRP, fasting glucose, insulin/HOMA, IGF-1, albumin, prealbumin 
3. Statistical analysis of the primary endpoint Absolute (mean [SD] and median [min/max]) scores for fatigue and HRQoL at inclusion and at each time point will be measured and compared between the two groups. An ANOVA will be carried out after determining the normality of the distribution of the scores. Univariate analyses of variance will be performed to compare the targeted dimensions at week 16 between the two groups, to calculate the average of the difference, and to identify potential confounders in the relationship between the exercise intervention, fatigue, and HRQoL. Factors with a statistical threshold of $5 \%$ will be integrated into a multivariate ANOVA. The results will be presented with $99 \%$ CI.

4. Statistical analysis of secondary endpoints TUDD scores for fatigue, targeted HRQoL dimensions, OS, and PFS will be estimated by the Kaplan-Meier method and compared using a log-rank test. The effect of the intervention will be expressed as hazard ratio (HR) with a 99 $\%$ CI. The Cox regression model will be used for multivariate analysis to measure the effect of the intervention on the five targeted dimensions. Different definitions of TUDD and types of events will be investigated in sensitivity analyses [20]. For the analysis of anxiety/depression and pain data, the variance analysis models will be used to assess whether, compared to baseline measurements, patients show changes in scores following the interventions (at each time point). Continuous data will be checked for normal distribution and, where applicable, log transformation will be applied to the data. The intraclass correlation coefficients for outcome variables of interest will be calculated. Univariate and multivariate logistic regression analyses of grade 3-4 toxicities and 3-4 late complications will be performed. Weight and BMI variables, as well as physical activity level and nutritional status variables, will be compared between groups. The HADS questionnaire will be completed by the physical activity partner and analyzed for descriptive purposes.

\section{Discussion}

Fatigue and altered HRQoL are common symptoms in patients with cancer receiving chemotherapy. Adapted physical activity during treatment is an original and promising non-pharmaceutical strategy that may help better manage these symptoms in supportive and palliative care interventions. Previous data have indicated that APA is feasible and efficient in various cancers. Effects of an exercise intervention in patients with advanced PDAC have never been explored. As these patients are strongly affected by fatigue, we hypothesized that they are likely to benefit from the exercise intervention. In addition, exercise has been reported to have a beneficial effect on tumor outcomes by reducing insulin resistance and insulin and IGF-1 secretion.
In this study, characteristics of patients with advanced PDAC that may affect the implementation of the APA program were taken into account. We propose an original method: the introduction of the physical activity partner to optimize patient adherence. This approach is based on the hypothesis that patient group sessions may be psychologically counterproductive in advanced PDAC patients because of the likely risk of short-term degradation of PS in several participants. Moreover, given that nutrition is a critical issue in the management of patients with PDAC and that physical exercise may increase patient denutrition by increasing energy expenditures, we carefully adapted a nutritional intervention to the APA program.

In conclusion, the APACaP study is a large multicenter randomized controlled trial to assess the feasibility and the efficacy of an unsupervised 16-week APA program on fatigue and HRQoL in patients with advanced PDAC. This intervention may appear challenging in those patients, given multiple cancer-related symptoms (fatigue, depression, pain, and denutrition). However, we hypothesize that the APA program may be beneficial for patients with advanced PDAC and may improve PDAC-related symptoms and HRQoL. If the APA program proves to be feasible and effective in this population, systematic implementation of this approach in association with anti-tumoral treatment will be the next logical step in this setting. Finally, the study results may also have implications for the multimodal treatment of other digestive cancers.

\section{Trial status}

APACaP started enrolling patients in September 2014. Study is ongoing, and the completion date is estimated at December 2017.

\section{Additional file}

Additional file 1: Table S1. Eastern Cooperative Oncology Group

(ECOG) Scale of Performance Status. (DOC 29 kb)

\section{Abbreviations}

1-RM: One-repetition maximum; ACS: American Cancer Society; ACSM: American College of Sports Medicine; AE: Adverse event AFSOS: Association Francophone pour les Soins Oncologiques de Support; ANSM: Agence Nationale de Sécurité du Médicament et des produits de santé; APA: Adapted physical activity; CA 19-9: carbohydrate antigen 19-9; CRP: C-reactive protein; CTCAE: Common Terminology Criteria for Adverse Events; ECOG: Eastern Cooperative Oncology Group; EORTC: European Organization for Research and Treatment of Cancer; FDR: Fixed-dose rate; FOLFIRINOX: 5-fluorouracil plus irinotecan plus oxaliplatin combination; GPAQ: Global Physical Activity Questionnaire; HADS: Hospital Anxiety and Depression Scale; HbA1c: glycated hemoglobin; HOMA: Homeostasis model assessment; HR: Hazard ratio; HRQoL: Health-related quality of life; IGF-1: Insulin-like growth factor 1; INCa: Institut National du Cancer; IPAQ: International Physical Activity Questionnaire; MFI-20: Multidimensional Fatigue Inventory; OS: Overall survival; PDAC: Pancreatic ductal adenocarcinoma; PFS: Progression-free survival; PS: Performance status; 
RECIST: Response evaluation criteria in solid tumors; RR: Relative risk; SD: Standard deviation; SFNEP: Société Francophone de Nutrition Clinique et Métabolisme; SHBG: Sex hormone binding globulin; TAP-CT: Thoraco-abdominopelvic computed tomography; TUDD: Time until definitive deterioration; WHO: World Health Organization.

\section{Competing interests}

The authors declare that they have no competing interests.

\section{Authors' contributions}

CN: study conception and design, manuscript drafting and revision. MV: study conception and design, manuscript revision. FB: study conception and design, manuscript revision. $\mathrm{PH}$ : study conception and design, manuscript revision. All authors have read and approved the final manuscript.

\section{Acknowledgments}

This work is funded by the ARCAD (Aide et Recherche en CAncérologie Digestive) foundation, with the support of the AREVA foundation and EY France (Ernst \& Young). The authors would like to thank Magdalena Benetkiewicz for editorial assistance in the preparation of the manuscript.

\section{Author details}

'Digestive Oncology Department and UMR1149, Beaujon University Hospital, Hôpitaux Universitaires Paris Nord Val de Seine (HUPNVS), Assistance Publique-Hôpitaux de Paris (AP-HP), 92110 Clichy La Garenne, France. ${ }^{2}$ Visio Activités Sportives Interactives (V@si SARL), 34270 Saint Mathieu de Tréviers, France. ${ }^{3}$ Methodology and Quality of Life in Oncology unit (EA 3181) and Quality of Life and Cancer Clinical Research Platform, Besançon University Hospital, 25000 Besançon, France.

\section{Received: 18 April 2015 Accepted: 29 September 2015} Published online: 12 October 2015

\section{References}

1. Wolin KY, Yan Y, Colditz GA, Lee IM. Physical activity and colon cancer prevention: a meta-analysis. Br J Cancer. 2009;100:611-6.

2. Harriss DJ, Atkinson G, Batterham A, George $K$, Cable NT, Reilly T, et al Lifestyle factors and colorectal cancer risk (2): a systematic review and meta-analysis of associations with leisure-time physical activity. Colorectal Dis. 2009;11:689-701

3. Friedenreich CM, Cust AE. Physical activity and breast cancer risk: impact of timing, type and dose of activity and population subgroup effects. $\mathrm{Br}$ J Sports Med. 2008:42:636-47.

4. Moore SC, Gierach GL, Schatzkin A, Matthews CE. Physical activity, sedentary behaviours, and the prevention of endometrial cancer. Br J Cancer. 2010;103:933-8.

5. Mustian KM, Sprod LK, Palesh OG, Peppone LJ, Janelsins MC, Mohile SG, et al. Exercise for the management of side effects and quality of life among cancer survivors. Curr Sports Med Rep. 2009:8:325-30.

6. Speck RM, Courneya KS, Masse LC, Duval S, Schmitz KH. An update of controlled physical activity trials in cancer survivors: a systematic review and meta-analysis. J Cancer Surviv. 2010;4:87-100.

7. Mishra SI, Scherer RW, Snyder C, Geigle PM, Berlanstein DR, Topaloglu O. Exercise interventions on health-related quality of life for people with cancer during active treatment. Cochrane Database Syst Rev. 2012:8:CD008465.

8. Cramp F, Byron-Daniel J. Exercise for the management of cancer-related fatigue in adults. Cochrane Database Syst Rev. 2012;11:CD006145.

9. Dimeo FC. Effects of exercise on cancer-related fatigue. Cancer. 2001;92:1689-93.

10. Velthuis MJ, Agasi-Idenburg SC, Aufdemkampe G, Wittink HM. The effect of physical exercise on cancer-related fatigue during cancer treatment: a metaanalysis of randomised controlled trials. Clin Oncol (R Coll Radiol). 2010;22:208-21.

11. Minton O, Richardson A, Sharpe M, Hotopf M, Stone P. Drug therapy for the management of cancer-related fatigue. Cochrane Database Syst Rev 2010; CD006704.

12. Courneya KS, Segal RJ, Mackey JR, Gelmon K, Reid RD, Friedenreich CM, et al. Effects of aerobic and resistance exercise in breast cancer patients receiving adjuvant chemotherapy: a multicenter randomized controlled trial. J Clin Oncol. 2007;25:4396-404.
13. Ballard-Barbash R, Friedenreich CM, Courneya KS, Siddiqi SM, McTiernan A, Alfano CM. Physical activity, biomarkers, and disease outcomes in cancer survivors: a systematic review. J Natl Cancer Inst. 2012;104:815-40.

14. Friedenreich CM, Woolcott CG, McTiernan A, Ballard-Barbash R, Brant RF, Stanczyk FZ, et al. Alberta physical activity and breast cancer prevention trial: sex hormone changes in a year-long exercise intervention among postmenopausal women. J Clin Oncol. 2010;28:1458-66.

15. Ligibel JA, Campbell N, Partridge A, Chen WY, Salinardi T, Chen H, et al. Impact of a mixed strength and endurance exercise intervention on insulin levels in breast cancer survivors. J Clin Oncol. 2008;26:907-12.

16. Massoner $\mathrm{P}$, Ladurner-Rennau M, Eder IE, Klocker H. Insulin-like growth factors and insulin control a multifunctional signalling network of significant importance in cancer. Br J Cancer. 2010;103:1479-84.

17. de Salles BF, Simao R, Fleck SJ, Dias I, Kraemer-Aguiar LG, Bouskela E. Effects of resistance training on cytokines. Int J Sports Med. 2010;31:441-50.

18. Kruijsen-Jaarsma M, Revesz D, Bierings MB, Buffart LM, Takken T. Effects of exercise on immune function in patients with cancer: a systematic review. Exerc Immunol Rev. 2013;19:120-43.

19. Quinten C, Coens C, Mauer M, Comte S, Sprangers MA, Cleeland C, et al. Baseline quality of life as a prognostic indicator of survival: a meta-analysis of individual patient data from EORTC clinical trials. Lancet Oncol. 2009;10:865-71.

20. Bonnetain F, Dahan L, Maillard E, Ychou M, Mitry E, Hammel P, et al. Time until definitive quality of life score deterioration as a means of longitudinal analysis for treatment trials in patients with metastatic pancreatic adenocarcinoma. Eur J Cancer. 2010:46:2753-62.

21. Wolin KY, Schwartz AL, Matthews CE, Courneya KS, Schmitz KH. Implementing the exercise guidelines for cancer survivors. J Support Oncol. 2012;10(5):171-7.

22. Schmitz KH, Courneya KS, Matthews C, Demark-Wahnefried W, Galvão DA, Pinto BM, et al. American College of Sports Medicine roundtable on exercise guidelines for cancer survivors. Med Sci Sports Exerc. 2010;42:1409-26.

23. Rock CL, Doyle C, Demark-Wahnefried W, Meyerhardt J, Courneya KS, Schwartz AL, et al. Nutrition and physical activity guidelines for cancer survivors. CA Cancer J Clin. 2012;62:242-74.

24. Buffart LM, Galvao DA, Brug J, Chinapaw MJ, Newton RU. Evidence-based physical activity guidelines for cancer survivors: current guidelines, knowledge gaps and future research directions. Cancer Treat Rev. 2014;40:327-40.

25. Hutzler $Y$, Sherrill C. Defining adapted physical activity: international perspectives. Adapt Phys Activ Q. 2007;24:1-20.

26. Jemal A, Bray F, Center MM, Ferlay J, Ward E, Forman D. Global cancer statistics. CA Cancer J Clin. 2011;61:69-90.

27. Ryan DP, Hong TS, Bardeesy N. Pancreatic adenocarcinoma. N Engl J Med. 2014;371:2140-1.

28. Reyes-Gibby CC, Chan W, Abbruzzese JL, Xiong HQ, Ho L, Evans DB, et al. Patterns of self-reported symptoms in pancreatic cancer patients receiving chemoradiation. J Pain Symptom Manage. 2007;34:244-52.

29. Muller-Nordhorn J, Roll S, Bohmig M, Nocon M, Reich A, Braun C, et al. Health-related quality of life in patients with pancreatic cancer. Digestion. 2006;74:118-25.

30. Mayr M, Schmid RM. Pancreatic cancer and depression: myth and truth. BMC Cancer. 2010;10:569

31. Maisonneuve P, Lowenfels AB. Epidemiology of pancreatic cancer: an update. Dig Dis. 2010;28:645-56.

32. Bao B, Wang Z, Li Y, Kong D, Ali S, Banerjee $\mathrm{S}$, et al. The complexities of obesity and diabetes with the development and progression of pancreatic cancer. Biochim Biophys Acta. 2011;1815:135-46.

33. O'Rorke MA, Cantwell MM, Cardwell CR, Mulholland HG, Murray L. Can physical activity modulate pancreatic cancer risk? A systematic review and meta-analysis. Int J Cancer. 2010;126:2957-68.

34. Dong X, Javle M, Hess KR, Shroff R, Abbruzzese JL, Li D. Insulin-like growth factor axis gene polymorphisms and clinical outcomes in pancreatic cancer. Gastroenterology. 2010;139:464-73. 473 e461-463.

35. Bergmann U, Funatomi H, Yokoyama M, Beger HG, Korc M. Insulin-like growth factor I overexpression in human pancreatic cancer: evidence for autocrine and paracrine roles. Cancer Res. 1995;55:2007-11.

36. Agustsson T, D'Souza MA, Nowak G, Isaksson B. Mechanisms for skeletal muscle insulin resistance in patients with pancreatic ductal adenocarcinoma. Nutrition. 2011;27:796-801. 
37. Greer JB, Whitcomb DC. Inflammation and pancreatic cancer: an evidence-based review. Curr Opin Pharmacol. 2009;9:411-8.

38. Fearon $\mathrm{K}$, Arends J, Baracos V. Understanding the mechanisms and treatment options in cancer cachexia. Nat Rev Clin Oncol. 2013;10:90-9.

39. Husebo AM, Karlsen B, Allan H, Søreide JA, Bru E. Factors perceived to influence exercise adherence in women with breast cancer participating in an exercise programme during adjuvant chemotherapy: a focus group study. J Clin Nurs. 2015;24:500-10.

40. Regan TW, Lambert SD, Girgis A, Kelly B, Kayser K, Turner J. Do couple-based interventions make a difference for couples affected by cancer? A systematic review. BMC Cancer. 2012;12:279.

41. Moses AW, Slater C, Preston T, Barber MD, Fearon KC. Reduced total energy expenditure and physical activity in cachectic patients with pancreatic cancer can be modulated by an energy and protein dense oral supplement enriched with n-3 fatty acids. Br J Cancer. 2004;90:996-1002.

42. Von Hoff DD, Ervin T, Arena FP, Chiorean EG, Infante J, Moore M, et al. Increased survival in pancreatic cancer with nab-paclitaxel plus gemcitabine. N Engl J Med. 2013;369:1691-703

43. Conroy T, Desseigne F, Ychou M, Bouché O, Guimbaud R, Bécouarn Y, et al. FOLFIRINOX versus gemcitabine for metastatic pancreatic cancer. N Engl J Med. 2011:364:1817-25.

44. Hammel P, Huguet F, van Laethem JL, Goldstein D, Glimelius B, Artru P, et al. Comparison of chemoradiotherapy (CRT) and chemotherapy (CT) in patients with a locally advanced pancreatic cancer (LAPC) controlled after 4 months of gemcitabine with or without erlotinib: final results of the international phase III LAP 07 study. J Clin Oncol. 2013;31:abstr LBA4003.

45. Eisenhauer EA, Therasse P, Bogaerts J, Schwartz LH, Sargent D, Ford R, et al. New response evaluation criteria in solid tumours: revised RECIST guideline (version 1.1). Eur J Cancer. 2009:45:228-47.

46. Aboodarda SJ, George J, Mokhtar AH, Thompson M. Muscle strength and damage following two modes of variable resistance training. J Sports SC Med. 2011;10:635-42.

47. Colado JC, Triplett NT. Effects of a short-term resistance program using elastic bands versus weight machines for sedentary middle-aged women. Strength Cond Res. 2008;22:1441-8.

48. Brzycki M. Strength testing - predicting a one-rep max from reps-tofatigue. JOPERD. 1997;64:88-90.

49. Page PA, Labbe A, Topp RV. Clinical force production of TheraBand ${ }^{\oplus}$ elastic bands. JOSPT. 2000;30:A47.

50. Smets EM, Garssen B, Bonke B, De Haes JC. The Multidimensional Fatigue Inventory (MFI) psychometric qualities of an instrument to assess fatigue. J Psychosom Res. 1995;39:315-25.

51. Gentile S, Delaroziere JC, Favre F, Sambuc R, San Marco JL. Validation of the French 'multidimensional fatigue inventory' (MFI 20). Eur J Cancer Care (Engl). 2003;12:58-64

52. Purcell A, Fleming J, Bennett $S$, Burmeister B, Haines T. Determining the minimal clinically important difference criteria for the Multidimensiona Fatigue Inventory in a radiotherapy population. Support Care Cancer. 2010;18:307-15

53. Aaronson NK, Ahmedzai S, Bergman B, Bullinger M, Cull A, Duez NJ, et al The European Organization for Research and Treatment of Cancer QLQ-C30: a quality-of-life instrument for use in international clinical trials in oncology. J Natl Cancer Inst. 1993;85:365-76.

54. Osoba D, Rodrigues G, Myles J, Zee B, Pater J. Interpreting the significance of changes in health-related quality-of-life scores. J Clin Oncol. 1998;16:139-44

55. Anota A, Hamidou Z, Paget-Bailly S, Chibaudel B, Bascoul-Mollevi C, Auquier $P$, et al. Time to health-related quality of life score deterioration as a modality of longitudinal analysis for health-related quality of life studies in oncology: do we need RECIST for quality of life to achieve standardization? Qual Life Res. 2015;24:5-18

56. Bonnetain F, Bonsing B, Conroy T, Dousseau A, Glimelius B, Haustermans K, et al. Guidelines for time-to-event end-point definitions in trials for pancreatic cancer. Results of the DATECAN initiative (Definition for the Assessment of Time-to-event End-points in CANcer trials). Eur J Cancer 2014:50:2983-93.

57. Cleeland CS, Ryan KM. Pain assessment: global use of the Brief Pain Inventory. Ann Acad Med Singapore. 1994;23:129-38.

58. Poundja J, Fikretoglu D, Guay S, Brunet A. Validation of the French version of the brief pain inventory in Canadian veterans suffering from traumatic stress. J Pain Symptom Manage. 2007;33:720-6.
59. Zigmond AS, Snaith RP. The hospital anxiety and depression scale. Acta Psychiatr Scand. 1983;67:361-70.

60. Razavi D, Delvaux N, Farvacques C, Robaye E. Validation of the French version of the Hospital Anxiety and Depression Scale (HADS) in a population of hospitalized cancer patients. Revue de Psychologie Appliquée. 1989;39:295-307.

\section{Submit your next manuscript to BioMed Central and take full advantage of:}

- Convenient online submission

- Thorough peer review

- No space constraints or color figure charges

- Immediate publication on acceptance

- Inclusion in PubMed, CAS, Scopus and Google Scholar

- Research which is freely available for redistribution 